\title{
Comments
}

\section{Comments on "A New Family of Cayley Graph Interconnection Networks of Constant Degree Four"}

\author{
Guihai Chen and Francis C.M. Lau, Member, IEEE
}

\begin{abstract}
Vadapalli and Srimani [2] have proposed a new family of Cayley graph interconnection networks of constant degree four. Our comments show that their proposed graph is not new but is the same as the wrap-around butterfly graph. The structural kinship of the proposed graph with the de Bruijn graph is also discussed.
\end{abstract}

Index Terms - Interconnection network, Cayley graph, generator, de Bruijn graph, butterfly graph, isomorphism.

\section{Definition OF Graph $G(n)$}

WE first give the definition of the graph $G(n)$ proposed by Vadapalli and Srimani [2].

Each node of $G(n)$ is represented as a circular permutation of $n$ different symbols in lexicographic order, where the $n$ symbols are presented in either uncomplemented or complemented form. Let $t_{k}, 0 \leq k \leq n-1$, denote the $k$ th symbol in the set of $n$ symbols. We use the English alphabet for the symbols: thus, for $n=4, t_{0}=a, t_{1}=b$, $t_{2}=c$, and $t_{3}=d$. We use $t_{k}^{*}$ to denote either $t_{k}$ or $\bar{t}_{k}$. Therefore, for $n$ distinct symbols, there are exactly $n$ different cyclic permutations of the symbols in lexicographic order, and, since each symbol can be present in either uncomplemented or complemented form, the node set of $G(n)$ has a cardinality of $n \times 2^{n}$. Since each node is some cyclic permutation of the $n$ symbols in lexicographic order, then, if $a_{0} a_{1} \ldots a_{n-1}$ denotes the label of an arbitrary node and $a_{0}=t_{k}^{*}$ for some integer $k$, then, for all $i, 1 \leq i \leq n-1$, we have $a_{i}=t_{(k+i)(\bmod n)}^{*}$. Thus, the definition of $G(n)$ is given as follows.

DEFINITION 1. The graph $G(n)$ is a Cayley graph whose nodes comprise the $n \times 2^{n}$ cyclic permutations of $n$ distinct symbols in lexicographic order. Each symbol is presented in either uncomplemented or complemented form. Given a node represented as a string $a_{0} a_{1} \ldots a_{n-1}$, its edges are defined by the following generators:

$$
\begin{gathered}
g\left(a_{0} a_{1} \ldots a_{n-1}\right)=a_{1} a_{2} \ldots a_{n-1} a_{0} \\
f\left(a_{0} a_{1} \ldots a_{n-1}\right)=a_{1} a_{2} \ldots a_{n-1} \bar{a}_{0} \\
g^{-1}\left(a_{0} a_{1} \ldots a_{n-1}\right)=a_{n-1} a_{0} \ldots a_{n-2} \\
f^{-1}\left(a_{0} a_{1} \ldots a_{n-1}\right)=\bar{a}_{n-1} a_{0} \ldots a_{n-2} .
\end{gathered}
$$

If the identity permutation is $t_{0} t_{1} \ldots t_{n-1}$, then the generator set $\Omega=\left\{f, g, f^{-1}, g^{-1}\right\}$ is given as:

\footnotetext{
- The authors are with the Department of Computer Science, The University of Hong Kong, Pokfulam Road, Hong Kong.

E-mail: \{gchen, fcmlau\}@cs.hku.hk.
}

Manuscript received 7 Dec. 1996; revised 8 Aug. 1997.

For information on obtaining reprints of this article, please send e-mail to: tpds@computer.org, and reference IEEECS Log Number 100356.

$$
\begin{gathered}
g=t_{1} t_{2} \ldots t_{n-1} t_{0} \\
f=t_{1} t_{2} \ldots t_{n-1} \bar{t}_{0} \\
g^{-1}=t_{n-1} t_{0} \ldots t_{n-2} \\
f^{-1}=\bar{t}_{n-1} t_{0} \ldots t_{n-2} .
\end{gathered}
$$

Fig. 1a shows $G(3)$ drawn in a "regular" fashion, which is different from that in [2]. The identity permutation of $G(3)$ is $a b c$, and the generator set is $\{b c a, b c \bar{a}, c a b, \bar{c} a b\}$. The nodes of $G(n)$ are grouped into different columns according to the position of the first symbol $t_{0}^{*}$ in their labels. In Fig. 1a, nodes with the symbol $a$ in the leftmost position of their labels form the first column, nodes with the symbol $a$ in the rightmost position form the second column, and nodes with the symbol $a$ in the middle position form the third column. The first column is duplicated in order to give a clearer view of the connections. We use solid lines to denote the $g$-edges, i.e., the edges defined by the permutation $g$ or $g^{-1}$, and dotted lines to denote the $f$-edges.

\section{ISOMORPHISM TO THE WRAP-AROUND BUTTERFLY GRAPH}

In this section, we prove that the graph $G(n)$ is isomorphic to the wrap-around butterfly graph $\mathcal{B}(n)$.

DEFINITION 2. The wrap-around butterfly graph $\mathcal{B}(n)$ has node-set $Z_{n} \times Z_{2}^{n}$. Each node is represented as a pair $\langle c, r\rangle$, where $c \in Z_{n}$ is the column of the node and $r \in Z_{2}^{n}$ is the row of the node. The edges of $\mathcal{B}(n)$ form butterflies (i.e., copies of the complete bipartite graph $\mathcal{K}_{2,2}$ ) between consecutive columns of nodes. Each node $\langle c, r\rangle$ is connected to the node $\left\langle c^{\prime}, r\right\rangle$ and the node $\left\langle c^{\prime}, r^{\prime}\right\rangle$, where $c^{\prime}=c+$ $1(\bmod n)$ and $r^{\prime}$ and $r$ differ in precisely the cth bit; the first edge is a straight edge and the second edge is a cross edge.

Fig. 1c shows $\mathcal{B}(3)$.

An isomorphical mapping between $\mathcal{G}(n)$ and $\mathcal{B}(n)$ is as follows: Given an arbitrary node $a_{0} a_{1} \ldots a_{n-1}$ in $G(n)$ and $a_{k}=t_{0}^{*}$ for some $k$, the node $a$ becomes $a^{\prime}=a_{k} a_{k+1} \ldots a_{n-1} a_{0} \ldots a_{k-1}$ after $(n-k)$ $(\bmod n) g^{-1}$ operations. If we substitute a 0 for every uncomplemented symbol and a 1 for every complemented symbol in $a^{\prime}$, and let the resulting binary string be $r$, then node $a_{0} a_{1} \ldots a_{n-1}$ in $G(n)$ corresponds to node $\langle n-k(\bmod n), r\rangle$ in $\mathcal{B}(n)$. It is not difficult to see that this mapping is a bijection. Furthermore, the $g$-edges in $G(n)$ correspond to the direct edges in $\mathcal{B}(n)$, while the $f$-edges in $G(n)$ correspond to the cross edges of $\mathcal{B}(n)$. To see the latter, consider nodes $a=a_{0} a_{1} \ldots a_{n-1}$ and $b=a_{1} \ldots a_{n-1} a_{0}$ in $G(n) . a$ and $b$ are connected by a $g$-edge. According to the above mapping, a corresponds to the node $\langle n-k(\bmod n), r\rangle$ in $\mathcal{B}(n)$, where $n-k$ and $r$ are computed as in the above; since $b=g(a)$, $t_{k}^{*}=a_{k}$ is at position $k-1(\bmod n)$ in $b$, and, so, $b$ corresponds to the node $\langle n-k+1(\bmod n), r\rangle$; clearly, these two nodes in $\mathcal{B}(n)$ are connected by a direct edge, by the definition of $\mathcal{B}(n)$. A similar analysis can be applied to the mapping between an f-edge in $G(n)$ and a cross edge in $\mathcal{B}(n)$. 


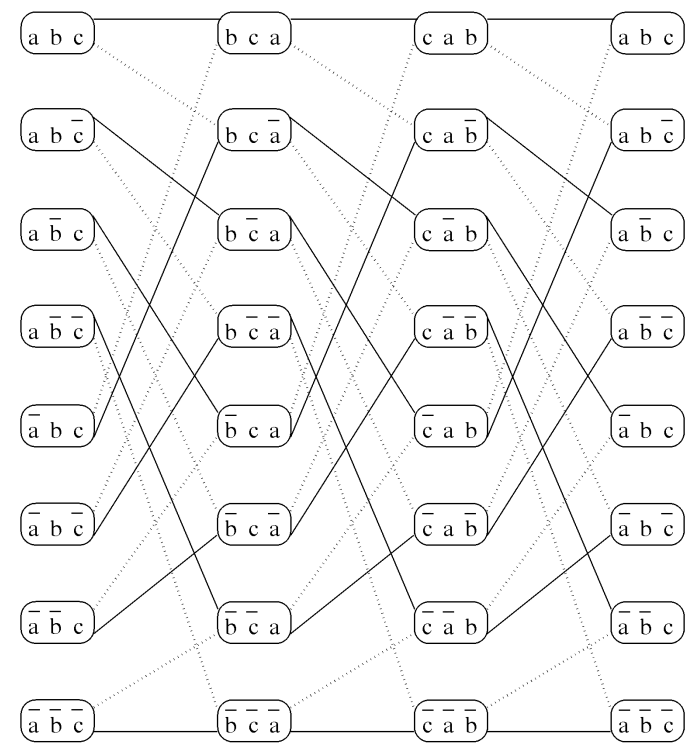

(a)

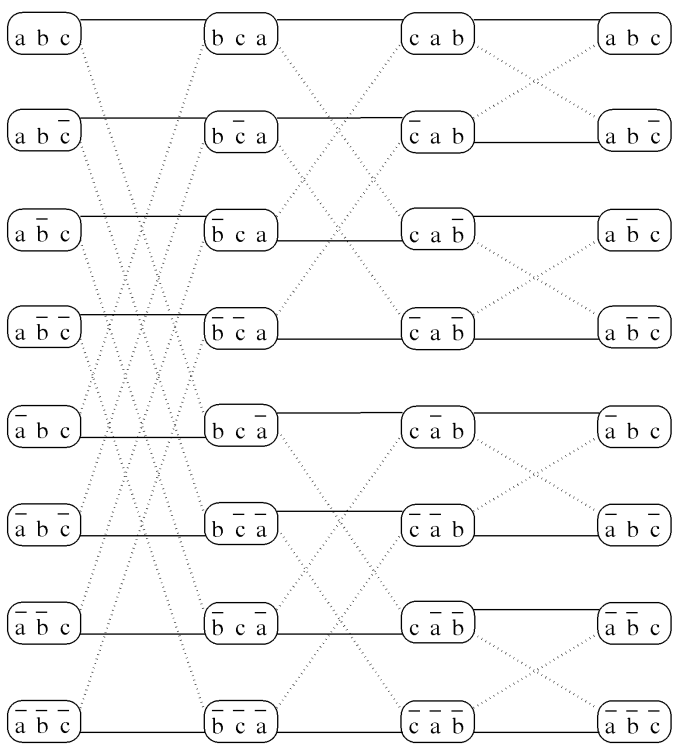

(b)

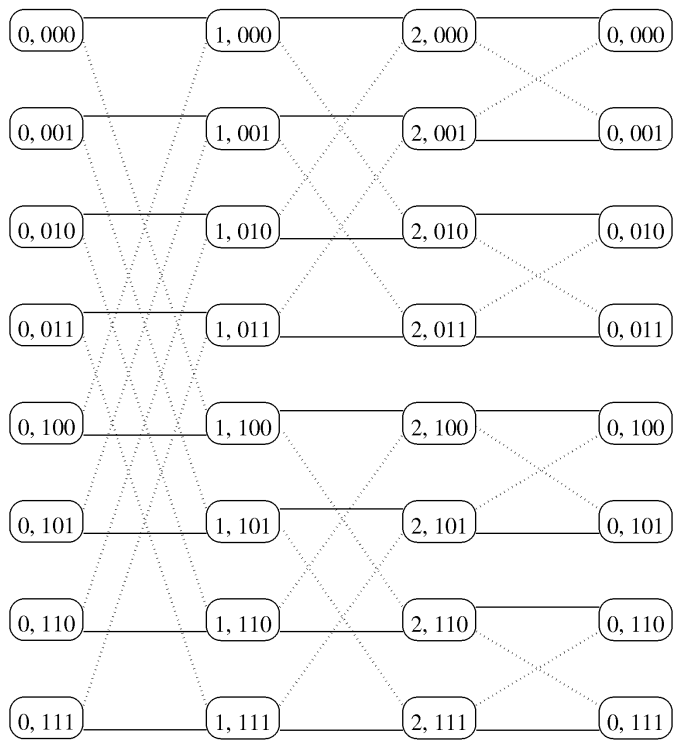

(c)

Fig. 1. (a) The proposed network, (b) after "straightening" the g-edges, (c) a wrap-around butterfly network. The solid lines are the g-edges in (a), (b), or the straight edges in (c); the dotted lines are the f-edges in (a), (b), or the cross edges in (c).

Refer again to Fig. 1 for an example. Based on the fact that a $g$ edge in $G(n)$ corresponds to a direct edge in $\mathcal{B}(n)$, we "straighten" all the g-edges in $\mathcal{G}(3)$ (Fig. 1a) (thus reordering the nodes in each column), and the result is the $G(3)$, as shown in Fig. 1b. Clearly, the latter is the same as the $\mathcal{B}(n)$ in Fig. 1c.

\section{FURTHER DISCUSSION}

We have shown that the graph $G(n)$ proposed by Vadapalli and Srimani is not a new graph, but a new representation of the wraparound butterfly graph. Indeed, $\mathcal{G}(n)=\mathcal{B}(n)$.

The group-theoretic relations between $\mathcal{B}(n)$ (or $G(n)$ ) and the de Bruijn graph are well studied in [1], where $\mathcal{B}(n)$ is proved to be a Cayley graph derived from the de Bruijn graph acting as a group action graph, and, inversely, the de Bruijn graph is proved to be some coset graph of $\mathcal{B}(n)$.
The new representation in [2] shows another simple structural kinship between $\mathcal{G}(n)$ (or $\mathcal{B}(n)$ ) and the de Bruijn graph. In particular, if $n$ distinct symbols in $G(n)$ are the same, i.e., each bit of the node address of $G(n)$ is either 0 or $1, G(n)$ specializes to become the de Bruijn graph.

The new representation in [2] may bring about some convenience in studying the topological properties of $\mathcal{G}(n)$ (or $\mathcal{B}(n))$, such as optimal routing algorithms and fault tolerance.

\section{REFERENCES}

[1] F. Annexstein, M. Baumslag and A.L. Rosenberg, "Group Action Graphs and Parallel Architectures," SIAM J. Computing, vol. 19, no. 3, pp. 544-569, June 1990.

[2] P. Vadapalli and P.K. Srimani, "A New Family of Cayley Graph Interconnection Networks of Constant Degree Four," IEEE Trans. Parallel and Distributed Systems, vol. 7, no. 1, pp. 26-32, Jan. 1996. 